\title{
Relation of Wertheim Association Constants to Concentration-based Equilibrium Constants for Mixtures with Chain-forming Components
}

\author{
Aseel M. Bala, Carl T. Lira \\ Department of Chemical Engineering \& Materials Science, Michigan State University, 2100 \\ Engineering Building, East Lansing, MI 48824, United States
}

Corresponding Author: Carl T. Lira, lira@egr.msu.edu

Submitted to Fluid Phase Equilibria, July 2016

\begin{abstract}
Several association modeling approaches have been developed to accurately describe the properties of polar solutions. Chemical theory and Wertheim's perturbation theory are among the most popular of these and they have been shown to yield similar functional forms for the contributions of association to Helmholtz energy and activity coefficients. In this paper, we study Flory polymerization theory through the work of Campbell and elucidate its correlation to Wertheim's theory. A simple key relationship between the concentration-based equilibrium constant and Wertheim's association constant is developed for systems in which all associating components have one acceptor and one donor site. Algebraic and numerical proofs are given for the equivalence of Flory's polymerization theory and Wertheim's perturbation theory for pure fluids and mixtures. Additionally, a new generalized activity expression is developed for Wertheim's theory.
\end{abstract}

\section{Keywords}

Association, Chemical theory, Perturbation theory, Wertheim theory 


\section{Introduction}

\subsection{Motivation}

As global demand for renewable chemicals and fuels increases, new pathways for their manufacture are being proposed. These projects include upgrading of natural oils, and fermentation of pyrolysis products to chemicals and fuels. The ubiquitous presence of polar components in biobased process streams complicates the design of industrial separation units. Traditional thermodynamic models, such as NRTL and UNIQUAC, are incapable of accurately representing liquid-liquid equilibria (LLE) and vapor-liquid-liquid equilibria (VLLE) in these systems without utilizing more parameters than can be determined with confidence of physical relevance [1].

The limitations of traditional models for polar systems are largely due to their crude representation of important molecular interactions; specifically, hydrogen bonding. Polar molecules hydrogen bond (or associate) with each other, forming clusters of chains and/or rings and causing large deviations from ideal behavior. These interactions are responsible for higher boiling points and enthalpies of vaporization of polar compounds compared to nonpolar molecules of comparable size. The extent and nature of association varies under different conditions including composition, temperature and pressure. Therefore, it is necessary for thermodynamic models to incorporate hydrogen bonding explicitly rather than lumping the contributions with nonpolar interactions.

\subsection{Historical Perspective}

Association models can be classified into three categories: chemical theories, lattice models and perturbation theories. Apelblat[2,3] presents a historical summary of the advancements made in association modeling from 1884 to 1984. Among the most noteworthy contributions is Dolezalek's[4] theory of representing a hydrogen bond as an equilibrium reaction governed by an equilibrium constant. The original theory, often called 'simple' or 'ideal' chemical theory, considers the true species formed through hydrogen bonding to otherwise behave ideally. In other words, physical interactions between the complexes in solution are ignored; a simplification that is difficult to justify and has proved erroneous for many systems especially in 
modeling liquid-liquid immiscibility. The Dolezalek body of work was therefore enhanced by adding a 'physical' contribution such as NRTL[5] or UNIQUAC[6].

With chemical theory, several decisions must be made a priori regarding the species that exist in solution and the equilibrium constants for various hydrogen bonds. The continuous association model, also known as the Mecke-Kempter model, makes the simplification that the value of the equilibrium constant does not depend on the chain or ring size formed. While there has been some discussion on the contradiction of this assumption with spectroscopic evidence[7], it is often adopted as a first-order representation of the complexation in solution.

Lattice models represent the system as a lattice of empty and occupied sites and investigate the extent of association by quantifying bonds between adjacent occupied sites. Perhaps the most prominent lattice model is the one presented by Guggenheim[8] which accounts for nonrandomness in the solution. Hydrogen bonding has since been integrated into several lattice models[9-12].

Perturbation theories add the association to a nonassociating reference fluid. Gubbins[13] recently presented a review of work done in this area. Several perturbation theories[14-16] have been developed but the simplest and most popular is Wertheim's theory[17-20], which is the basis of a number of equation of state (EOS) models including Elliott-Suresh-Donohue (ESD)[21,22], cubic plus association (CPA)[23,24] and the statistical associating fluid theory (SAFT) family of equations[25-30].

Numerous independent researchers [31-33] have compared association models. Economou and Donohue[31] found that for many systems, both chemical theory (using activity-based $K_{a}$ ) and Wertheim's theory yield essentially the same functional form for the association contribution, despite differences in their origins and derivations. Wolbach and Sandler derived relations between $K_{a}$ and Wertheim's association constant and used quantum calculations to determine values of the Wertheim association constant for molecules with multiple sites[32,34]. Chapman et al.[25] noticed the similarity in polymer distributions using Wertheim's theory in the SAFT model compared to Flory's polymerization theory (using concentration-based $K_{C}$ ). Campbell rearranged the Flory polymerization theory and was able to find a similar algebraic form but the terms were not identical [35]. Thus, the analytical equivalence of the Wertheim theory and Flory’s polymerization theory has not yet been proven. In this work, we compare the two 
approaches for systems of chain-forming species in which the associating components each have an acceptor site and a donor site to contribute to association. Examples of these systems include pure alcohols, alcohol + inerts and alcohol + alcohol mixtures. We also provide algebraic and numerical proofs of the equivalence of Flory's theory and Wertheim's theory when the packing factor is universal and the excess volume is zero.

\section{Theory and Analysis}

A nonideal system's behavior is described using either deviations from ideal gas mixtures using fugacity coefficients, or deviations from ideal solutions using activity coefficients as discussed by Prausnitz and Tavares[36]. Fugacity coefficients are readily calculated with an equation of state through the compressibility factor, $Z$. It is common practice to separate contributions to $Z$ according to the nature of the nonideality causing the deviations:

$$
Z-Z^{i g}=Z-1=Z^{r e p}+Z^{a t t}+Z^{a s s o c}
$$

where $Z^{r e p}$ and $Z^{a t t}$ represent effects of the repulsive and attractive forces and $Z^{a s s o c}$ accounts for hydrogen bonding between molecules. Contributions to the Helmholtz energy departure at fixed $T$ and $V$ are correspondingly:

$$
\left(A-A^{i g}\right)_{T, V}=A^{r e p}+A^{a t t}+A^{a s s o c}
$$

In a model such as the SAFT EOS, a bond term is added to both $Z-Z^{i g}$ and $\left(A-A^{i g}\right)_{T, V}$. An alternative approach is to consider the excess Gibbs energy $\left(G^{E}\right)$, related directly to the activity coefficient, $\gamma$, of each component and separate perturbations according to their sources. Deviations caused by differences in the size and shapes of molecules are accounted for by an entropic combinatorial contribution which ignores all attractive interactions and is the only term remaining at infinitely high temperatures. The association contribution represents nonidealities due to hydrogen bonding and the residual term accounts for other interactions that are not as well-understood and typically includes adjustable parameters. Combining all the contributions yields the activity coefficient:

$$
\ln \gamma=\ln \gamma^{c o m b}+\ln \gamma^{r e s}+\ln \gamma^{a s s o c}
$$

The focus of this work is the $\gamma^{a s s o c}$ term. We begin by introducing the nomenclature used to describe the apparent and true concentrations in associating solutions. Here, the term 'apparent' 
is used to denote 'stoichiometric' or 'superficial' species in a mixture. The speciation that occurs in solution results in 'true' species, which are model-dependent. For example, in a pure alcohol, the apparent mole fraction of alcohol is unity, but the solution can also be described in terms of concentrations of true monomers, dimers, trimers and so on. Notations vary in literature, and the distinction between apparent and true mole is not always clear. In this work, a subscript $T$ is used to denote the true quantities. Every solution will contain some 'free' or 'unassociated' molecular species which is described as monomer and denoted with the subscript $\mathrm{M}$, which is understood to be a 'true' quantity.

\subsection{Association term - Wertheim's Theory}

Wertheim's perturbation theory calculates the probabilities of various site interactions that result in the formation of a complex. Each molecule type in solution may have any number of acceptor $(A)$ and/or donor $(D)$ sites and bonding between each pair is governed by an association strength parameter, $\Delta^{A_{i} D_{j}}$. Notation for the acceptor and donor sites subscripts differs in literature, thus comparisons must be done carefully. In early literature, the $i$ and $j$ denote the host component. However, as noted by Michelsen[37], the summations are greatly simplified by indexing the sites rather than the site hosts because site hosts can have more than one type of each site. We write summations over sites in this work. The acceptor and donor terminology allows the model to lend itself to any type of complexation, including covalent bonding. Depending on the number and types of sites attributed to a molecule of a component, different association schemes can be used as reviewed by Kontogeorgis and Folas[38]. Alcohols, for example, are commonly modeled using the 2B association scheme which corresponds to having one acceptor and one donor site per molecule (Figure 1). We follow this convention in the present work. A detailed explanation of the assumptions and implications of Wertheim’s theory is given by Chapman[25].

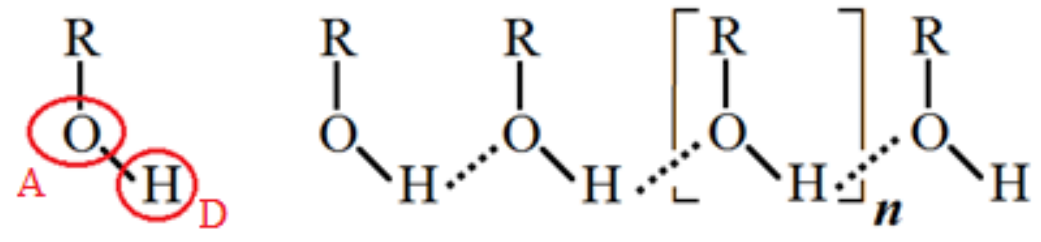

Figure 1: Hydrogen bonded oligomers with two bonding sites per molecule. The acceptor and donor sites are labeled on the leftmost molecule. 
The Helmholtz energy departure at fixed temperature and volume due to association derived from Wertheim's theory is implemented in SAFT, ESD and CPA as:

$$
\frac{\left(\underline{A}-\underline{A}^{i g}\right)_{T, V}^{\text {assoc }}}{R T}=\sum_{\begin{array}{c}
\text { all site } \\
\text { types } B_{i}
\end{array}} n_{0, B_{i} \text { host }} N_{B_{i}}\left(\ln X^{B_{i}}-\frac{X^{B_{i}}}{2}+\frac{1}{2}\right)
$$

where $B_{i}$ is generic notation for an acceptor, donor, or bivalent (acceptor/donor) site, $n_{0, B_{i} \text { host }}$ is the apparent number of moles of the component hosting site $B_{i}, N_{B_{i}}$ is the number of identical sites of type $B$ on the host. The term $X^{B_{i}}=n^{B_{i} \text { free }} / n^{B_{i} \text {,total }}$ is the fraction of sites of type $B$ that

$$
X^{B_{i}}=\left(1+\sum_{\substack{\text { mating } \\ \text { sites } E_{j}}} x_{E_{j} \text { host }} N_{E_{j}} X^{E_{j}} \rho \Delta^{B_{i} E_{j}}\right)^{-1}
$$

remain nonbonded at equilibrium, calculated through the balance on the probability of binding:

where $E_{j}$ is generic notation for a site which mates with site $B_{i}, x_{E_{j}}$ host denotes the component apparent mole fraction of the site $E_{j}$ host and $\Delta^{B_{i} E_{j}}$ is an association strength parameter between site $i$ of type B and site $j$ of type $E$. In this work $\rho$ is molar density and thus $\Delta^{B_{i} E_{j}}$ has units of molar volume.

With chain-forming components, when each hydrogen bond formed, one acceptor $(A)$ and one donor $(D)$ site are consumed. Thus, in a pure fluid with the 2B association scheme, the fraction of molecules nonbonded at each acceptor site is the same as those free at the donor sites.

$$
X^{A_{i}}=X^{D_{i}}
$$

Therefore, numbering acceptor and donor sites on the same host using the same subscripts,

For mixtures of associating species with the 2B model, Eq. (6) is contingent on $\Delta^{A_{i} D_{j}}=\Delta^{D_{j} A_{i}}$, which is true when certain cross-coefficients equations are used. The reader should recognize that site indexes $i$ and $j$ match the host indexes for the 2B model indexing discussed here. The 
combining rules we use to calculate cross-coefficients are consistent with Eq. (6) and are discussed in Section 2.6.

To derive a formula for $\gamma_{i}^{\text {assoc }}$ using Wertheim's theory, we consider the work of Michelsen and Hendriks[39] in deriving a generalized function which reduces to Eq. (4) when maximized and results in the association contribution to chemical potential. This approach is extended to calculate the fugacity coefficient of each component in the mixture in Elliott and Lira[40] (Important corrections are made in the errata[41]). Calculating the activity coefficient from the fugacity coefficient for a given component (Appendix A) for the case of conventional mixing

$$
\ln \gamma_{k}^{\text {assoc }}=\sum_{\substack{\text { sites } B_{i} \\ \text { on } k}} N_{B_{i}}\left[\ln \left(\frac{X^{B_{i}}}{X^{B_{i}, 0}}\right)-\frac{1}{2}\left(1-X^{B_{i}, 0}\right)\right]+\frac{1}{2} \frac{\rho}{\rho_{k}} \sum_{\substack{\text { all } \\ \text { sites } B_{i}}} x_{B_{i} \text { host }} N_{B_{i}}\left(1-X^{B_{i}}\right)
$$

rules, van der Waals $Z^{r e p}$, a universal packing factor, and no excess volume yields:

where $X^{B_{i}}$ is given by Eq. (5), and $X^{B_{i}, 0}$ represents the fraction of sites free when the host for the site is pure. These constraints are identical to those used in Flory's polymerization theory. This activity coefficient equation is easier to apply than the equation of Fu and Sandler [42] because derivatives of $X^{B_{i}}$ are not needed. A more general expression is derived by substituting Eq. (A10) of Appendix A into Eq. (A14).

\subsection{Flory's Concentration-based Equilibrium Constant}

In chemical theory, the energetic effects associated with hydrogen bonding are embedded within an equilibrium constant, $K$. This parameter could be based on the concentrations $\left(K_{C}\right)$, mole fractions $\left(K_{x}\right)$ or activities $\left(K_{a}\right)$ of the products and reactants. Flory's well-known work on oligomer modeling used a concentration-based equilibrium constant and provides a discussion on the inconsistency of $K_{x}$ with statistical mechanics[43]. Flory's original form of $K_{C}$ and variations of it have since been applied in several publications. Chemical kinetics are commonly described in practice using concentrations, and for reversible reactions are consistent with $K_{C}$, which is frequently assumed to be independent of composition. This simplification is valid only when noncombinatorial effects are invariant with composition. For pure alcohols or alcohol-inert systems, some of the variations of $K_{C}$ for the formation of a chain of size $i+1$ from a complex of size $i$ and a monomer are shown in Table 1. 
Table 1: Variations of the concentration based equilibrium constant used in literature. The subscript numeral on $K_{C}$ differentiates between models.

\begin{tabular}{|c|c|c|}
\hline $\boldsymbol{K}_{\boldsymbol{C}}$ & References & Eqn \\
\hline$K_{C 1}=\frac{C_{T, i+1}}{C_{T, i} C_{M}}=\frac{x_{T, i+1}}{x_{T, i} x_{M}} \frac{1}{\rho_{T}}$ & $\begin{array}{c}\text { Flory[43], Renon and } \\
\text { Prausnitz[44,45] (eqn. 2) }\end{array}$ & 8 \\
\hline$K_{C 2}=\frac{\Phi_{T, i+1}}{\Phi_{T, i} \Phi_{\mathrm{M}}} \frac{i}{i+1}=\frac{x_{T, i+1}}{x_{T, i} x_{M}} \frac{\rho_{A}}{\rho_{T}}=K_{C 1} \rho_{A}$ & $\begin{array}{c}\text { Flory[43], Nagata[46], Nath and } \\
\text { Bender[47], Renon and } \\
\text { Prausnitz[44,45] (eqn. 1) }\end{array}$ & 9 \\
\hline$K_{C 3}=\frac{\Phi_{T, i+1}}{\Phi_{T, i} \Phi_{\mathrm{M}}}=\frac{x_{T, i+1}}{x_{T, i} x_{M}} \frac{\rho_{A}}{\rho_{T}} \frac{i+1}{i}=K_{C 1} \rho_{A} \frac{i+1}{i}$ & Nagata[48] & 10 \\
\hline$K_{C 4}=\frac{\Phi_{T, i+1}}{\Phi_{T, i} \Phi_{\mathrm{M}}} \frac{i r_{A}}{i+1}=\frac{x_{T, i+1}}{x_{T, i} x_{T, M}} \frac{\rho^{r e f}}{\rho_{T}}=K_{C 1} \rho^{r e f}$ & Brandani[49,50], Campbell[35] & 11 \\
\hline
\end{tabular}

Here, $\rho_{A}$ represents the pure alcohol molar density. $\Phi_{i}$ is the volume fraction. The variable $\rho_{T}=n_{T} / \underline{V}$, is the true molar density which is smaller than the apparent molar density $\rho=n_{0} / \underline{V}$, because bonding reduces the number of moles, $n_{T} \leq n_{0}$. In Eq. (11), $r_{A}$ is a measure of the molecular size of the associating species relative to some reference $\left(r_{A}=\rho^{r e f} / \rho_{A}\right)$, chosen by Campbell to be the molar density of methanol at $303.15 \mathrm{~K}$.

\subsection{Relationship between $K_{a}, K_{C}$ and $\Delta$}

To understand the relationship between $K_{a}$ and $K_{C}$, we use equations for a pure component with one acceptor and one donor. These are later extended to mixtures using combining rules in section 2.6. We begin with the thermodynamic definition of the activity based equilibrium constant:

$$
K_{a}=\frac{x_{T, i+1}}{x_{T, i} x_{T, M}} \frac{\hat{\varphi}_{i+1}}{\hat{\varphi}_{i} \hat{\varphi}_{M}} \frac{P^{\circ}}{P}
$$

where $P^{\circ}$ is the standard state pressure, typically 1 bar. Inserting Eq. (8) into Eq. (12) yields:

$$
K_{a}=K_{C 1} \rho_{T} \frac{\hat{\varphi}_{i+1}}{\hat{\varphi}_{i} \hat{\varphi}_{M}} \frac{P^{\circ}}{P}
$$

Economou and Donohue[31] discuss different conventions used for equilibrium constants. By comparing the monomer fraction derived from various models, they conclude that $\Delta$ must be 
proportional to $K_{a}$ at the same $T$. Wolbach and Sandler[32] explore the relationship further and

$$
\Delta^{\mathrm{ig}}=f K_{a} \frac{R T}{P^{\circ}}
$$

show that in the case where the vapor phase is ideal:

where $f$ is a constant that depends on the association scheme used to model the component in solution. For liquid components modeled with 2B, Eq. (14) becomes:

$$
\Delta=e^{g} K_{a} \frac{R T}{P^{\circ}}
$$

where $g$ is a function that depends on the equation of state used to calculate the fugacity coefficient ratio. The value of this function depends on the mixing rules used for the true solution of monomers and oligomers. The most accepted and widely used combining rules for complexes, introduced by Heidemann and Prausnitz[51], are:

$$
\begin{gathered}
a_{i}=i^{2} a_{M} \\
b_{i}=i b_{M}
\end{gathered}
$$

where $a_{M}$ and $b_{M}$ are the interaction and size parameters for monomers respectively. Heidemann and Prausnitz[51] show that when Eqs. (16) and (17) and the Lorentz-Berthelot mixing rules

$$
g=\int_{0}^{\eta} \frac{Z^{r e p}}{\eta} d \eta
$$

( $b=\sum_{j} x_{j} b_{j}, a=\sum_{j} \sum_{k} x_{j} x_{k} a_{j k}$ ) are followed, then $g$ is given by Eq. (18)

From this equation, an important observation can be made. While $K_{a}$ depends only on temperature, Wertheim's association strength $\Delta$ of Eq. (15) is also a function of the density of the solution due to the $g$ function. Kontogeorgis and Folas[38] summarized the assumptions taken in various models in literature and the resulting forms of $g$. When the van der Waals repulsive term is used, Eq. (18) gives the radial distribution function at contact distance, $g(\sigma)$. Moreover, applying the combining rules (Eqs. (16) and (17)) to the van der Waals equation for the component fugacity coefficients provides a useful relationship between the fugacity coefficient and true compressibility factor as shown in Appendix B. Calculating the ratio of the fugacity 
coefficients for the reaction of a complex of size $i$ and a monomer to form a complex of size $i+1$ yields:

$$
\ln \left(\frac{\hat{\varphi}_{i} \hat{\varphi}_{M}}{\hat{\varphi}_{i+1}}\right)=g(\sigma)-\ln Z_{T}
$$

or

$$
e^{g(\sigma)}=\frac{P \underline{V}}{n_{T} R T} \frac{\hat{\varphi}_{i} \hat{\varphi}_{M}}{\hat{\varphi}_{i+1}}
$$

Substituting Eq. (20) into Eq. (15), then converting to apparent density and inserting Eq. (13):

$$
\Delta=\frac{P \underline{V}}{P^{\circ} n_{T}} \frac{\hat{\varphi}_{i} \hat{\varphi}_{M}}{\hat{\varphi}_{i+1}} K_{a}=\frac{P K_{a}}{P^{\circ} \rho_{0}} \frac{\hat{\varphi}_{i} \hat{\varphi}_{M}}{\hat{\varphi}_{i+1}} \frac{n_{0}}{n_{T}}=\frac{K_{C 1} \rho_{T}}{\rho_{0}} \frac{n_{0}}{n_{T}}
$$

Recognizing that $\rho_{T} / \rho_{0}=\left(n_{T} / \underline{V}\right) /\left(n_{0} / \underline{V}\right)=n_{T} / n_{0}$ yields a remarkably simple $\Delta-K_{C}$ relationship for pure fluids:

$$
\Delta=K_{C 1}
$$

This result is intuitive considering the conclusions arrived at by several investigators[31,33,39] on the mathematical similarity of chemical and Wertheim's theories. However, it is powerful in that it allows one to use and compare both association models with ease.

\subsection{Association term - Campbell's Chemical Theory Model}

Pradhan et al.[52] developed a chemical theory model by integrating an equilibrium constant into the Flory-Huggins (FH) theory. Campbell[35] extended the expression to systems containing any number of alcohols. In this section, we clarify the relationship between this set of equations and other association models.

$$
\ln \gamma_{i}^{\text {chem }}=\ln \left(\frac{C_{M_{i}}^{\prime}}{x_{i} C_{M_{i}}^{\prime 0}}\right)-r_{i}\left(\Psi-\Psi_{i}^{0}\right)
$$

Following Flory[43], Campbell writes:

where $C^{\prime}{ }_{M_{i}}$ and $C^{\prime 0}{ }_{M_{i}}$ are the dimensionless true monomer concentration of component $i$ in a mixture and at purity, respectively. All concentrations, $C^{\prime}$, are made dimensionless by dividing 
by the liquid density of methanol at 303.15. $\Psi$ is the total molar concentration of the true

$$
\Psi=\sum_{i} \Psi_{i} ; \quad \Psi_{i}=\frac{C_{i}^{\prime}}{1+\sum_{j} K_{C 4, j i} \Psi_{j}}
$$

complexes in solution which Campbell shows is given by:

where the summations are over apparent species and $C^{\prime}{ }_{i}$ is the apparent dimensionless concentration of component $i$. As shown in Appendix C, Campbell's $\Psi_{i}$ can be recognized as the total molar concentration of species with a free end group $i$.

The Flory concentration-based $K_{C 4, j i}=C_{T(j, n+1)}^{\prime} /\left(C_{T(i, n)}^{\prime} C_{T(j, 1)}^{\prime}\right)$ is the equilibrium constant for the association between a chain of length $n$ with a nonbonded acceptor $i$ forming a bond with monomer $j$ donor, resulting in a chain of length $n+1$ with a nonbonded $j$ acceptor end. Campbell does not discuss acceptors or donors, but the nomenclature helps clarify how, when the alcohol monomer donor bonds to an end of a chain, it can still participate through its free acceptor. Note that the nomenclature for acceptors and donors could be flipped without changing the balances. Campbell provides mathematical derivations for Eqs. (23) and (24). The free end on a chain could be an acceptor or a donor. Considering the end to be an acceptor, $\Psi_{i}$ represents the dimensionless molar concentration of nonbonded acceptors of type $i$. Recognizing that, because each alcohol molecule possesses one acceptor site, the dimensionless apparent molar concentration of all acceptors on component $i$ in solution is equal to $C^{\prime}{ }_{i}$. Therefore, the fraction of

$$
\frac{\Psi_{i}}{C^{\prime}}=\frac{n^{A_{i}}}{n^{A_{i}, T}}=X^{A_{i}}
$$

unbonded acceptors is or $\Psi_{i}=C^{\prime}{ }_{i} X^{A_{i}}$. Substituting the dimensionless concentration in terms of molar densities ${C^{\prime}}_{i}=$

$$
\Psi_{i}=x_{i} \rho X^{A_{i}} / \rho^{r e f}
$$

$\Phi_{i} \rho_{i} / \rho^{r e f}=x_{i} \rho / \rho^{r e f}$, we find that: 
Therefore, $\sum_{j} K_{C j i} \Psi_{j}$ can be written as $\sum_{j} x_{j} \rho K_{C 4, j i} X^{D_{j}} / \rho^{r e f}$. Simple manipulation will show that the right expression of Eq. (24) is the same as Eq. (5).

\subsection{Algebraic Equivalence of Chemical and Wertheim's Theory}

$$
\begin{array}{r}
\ln \gamma_{1}^{a s s o c}=2 \ln \left(\frac{X^{A}}{X^{A, 0}}\right)-\left(1-X^{A, 0}\right)+\frac{\rho}{\rho_{1}} x_{1}\left(1-X^{A}\right) \\
=2 \ln \frac{X^{A}}{X^{A, 0}}+X^{A, 0}-\left(1-\Phi_{1}\right)-X^{A} \Phi_{1} \\
\ln \gamma_{2}^{a s s o c}=\frac{\rho}{\rho_{2}} x_{1}\left(1-X^{A}\right)=\frac{\Phi_{2}}{x_{2}} x_{1}\left(1-X^{A}\right)
\end{array}
$$

For a binary alcohol (1) - inert (2) system, Wertheim’s theory (Eq. (7)) gives:

For the same system, we begin the analysis of Campbell's model by calculating $\Psi$ and $\Psi_{i}^{0}$ by combining Eqs. (24) and (26):

$$
\begin{gathered}
\Psi=\Psi_{1}+\Psi_{2}=\frac{x_{1} \rho X^{A}}{\rho^{r e f}}+\frac{x_{2} \rho}{\rho^{r e f}} \\
\Psi_{1}^{0}=\frac{\rho_{1} X^{A, 0}}{\rho^{r e f}} ; \Psi_{2}^{0}=\frac{\rho_{2}}{\rho^{r e f}}
\end{gathered}
$$

Next, we calculate the values of $C_{T, M_{i}}^{\prime}$ and $C_{T, M_{i}}^{\prime 0}$. Campbell provides the relationship between the concentration of monomers ending with group $i$ and $C^{\prime}{ }_{i}$ given as $C_{T, M_{i}}^{\prime}=\Psi_{i}^{2} / C^{\prime}{ }_{i}$. Substituting into this equation with the definition of $C^{\prime}{ }_{i}$ and Eq. (28), we find for both components in the mixture and at purity:

$$
\begin{aligned}
& C_{T, M_{1}}^{\prime}=\frac{x_{1} \rho}{\rho^{r e f}}\left(X^{A}\right)^{2} ; C_{T, M_{2}}^{\prime}=\frac{x_{2} \rho}{\rho^{r e f}} \\
& C_{T, M_{1}}^{\prime 0}=\frac{\rho_{1}}{\rho^{r e f}}\left(X^{A, 0}\right)^{2} ; C_{T, M_{2}}^{\prime 0}=\frac{\rho_{2}}{\rho^{r e f}}
\end{aligned}
$$

Therefore, by combining Eqs. (29-32), Eq. (23) for the alcohol becomes:

$$
\ln \gamma_{1}^{c h e m}=2 \ln \frac{X^{A}}{X^{A, 0}}+\ln \left(\frac{\Phi_{1}}{x_{1}}\right)-X^{A} \Phi_{1}-\frac{\Phi_{1}}{x_{1}}+\Phi_{1}+X^{A, 0}
$$

Adding and subtracting 1, and rearranging yields: 


$$
\begin{gathered}
\ln \gamma_{1}^{\text {chem }}=2 \ln \frac{X^{A}}{X^{A, 0}}+X^{A, 0}-\left(1-\Phi_{1}\right)-X^{A} \Phi_{1}+\ln \left(\frac{\Phi_{1}}{x_{1}}\right)+1-\frac{\Phi_{1}}{x_{1}} \\
=\ln \gamma_{1}^{\text {assoc }}+\ln \gamma_{1}^{\text {Flory }}
\end{gathered}
$$

where Flory's equation[43] for the combinatorial contribution to activity coefficients is

$$
\ln \gamma_{i}^{\text {Flory }}=\ln \left(\frac{\Phi_{i}}{x_{i}}\right)+1-\frac{\Phi_{i}}{x_{i}}
$$

Similarly, rearranging Eq. (23) and substituting Eqs. (29-32) for the inert solvent gives:

$$
\ln \gamma_{2}^{\text {chem }}=\frac{x_{1} \rho}{\rho_{2}}\left(1-X^{A}\right)+\ln \left(\frac{\Phi_{2}}{x_{2}}\right)+1-\frac{\Phi_{2}}{x_{2}}=\ln \gamma_{2}^{\text {assoc }}+\ln \gamma_{2}^{\text {Flory }}
$$

Thus, this provides analytical proof that Campbell's chemical theory incorporates combinatorial effects through Flory's equation. Consequently, when Wertheim's theory is compared to Campbell's model, the Flory term must be added separately. When calculating activity

$$
\ln \gamma_{i}^{\text {chem }}=\ln \gamma_{i}^{\text {assoc }}+\ln \gamma_{i}^{\text {Flory }}
$$

coefficients using Campbell's chemical theory, the combinatorial term is inherently included.

\subsection{Calculating Association Parameters}

To clarify the comparison further, we provide some numerical calculations. Early work done in determining $K_{C}$ for binary alcohol-inert systems used data reduction of mixture data to fit association constants[44,45]. Commonly, the value of $K_{c}$ is assumed to be independent of solvent species or concentration. Therefore, models based only on the properties of the pure associating compound were developed. Nath and Bender[47] based $K_{C}$ on the temperature and enthalpy of vaporization at the normal boiling point of the alcohol. Lobien[53] calculated smaller $K_{C}$ values than others using limiting activity coefficient data. Because these approaches differ considerably, there is significant scatter in the resulting $K_{C}$ values for the same alcohol [47]. For CPA and SAFT, the $\Delta^{A_{i} D_{j}}$ values are typically determined by fitting density and vapor pressure[23,25,54].

In this work, to provide numerical calculations, we follow the approach outlined by Nagata[46] in applying the Brandani[49]'s work. The model essentially assumes that the only differences between the vapor pressure of an alcohol and that of a nonassociating compound with an 
identical molecular weight and chemical formula, i.e. its homomorph, are the consequences of hydrogen bonding. Therefore, $K_{C}$ is determined by matching the two vapor pressures through Eq. (38).

$$
P_{A}^{v a p}=\gamma_{M}^{0} x_{M}^{0} P_{h}^{v a p} \exp \left(\frac{\left(P_{A}^{v a p}-P_{h}^{v a p}\right)\left(V_{A}^{L}-B_{A}^{f r e e}\right)}{R T}\right)
$$

where $B_{A}{ }^{\text {free }}$ is the free contribution to the second virial coefficient evaluated using Hayden and O'Connell's calculations[55] and $\gamma_{A M}^{0}$ is the activity coefficient of the alcohol monomer in pure alcohol as given by Flory's equation.

The volume and mole fractions of monomer in pure alcohol are calculated as[46]:

$$
\begin{gathered}
\Phi_{M}^{0}=\frac{1+2 K_{C 2}-\sqrt{1+4 K_{C 2}}}{2 K_{C 2}{ }^{2}} \\
x_{M}^{0}=1-K_{C 2} \Phi_{M}^{0}
\end{gathered}
$$

Ethers were selected as homomorphs for alcohols and their vapor pressures were determined using Ambrose's[56] correlation with the same $n *$ (effective number of carbon atoms in the ether homomorph) values as Nagata. Our resulting values of $K_{C 2}$ are given in Table 2.

Table 2: Equilibrium constant for primary alcohols at $50{ }^{\circ} \mathrm{C}$

\begin{tabular}{|c|c|c|}
\hline Alcohol & $\boldsymbol{K}_{\boldsymbol{C} \mathbf{2}}$ & $\boldsymbol{K}_{\boldsymbol{C 1}}=\boldsymbol{\Delta}$ \\
\hline Methanol & 111.0 & 4549 \\
\hline Ethanol & 81.48 & 4803 \\
\hline 1-Propanol & 71.27 & 5381 \\
\hline 1-Butanol & 69.88 & 6458 \\
\hline 1-Pentanol & 57.09 & 6233 \\
\hline
\end{tabular}

We have refitted the $K_{C 2}$ values to be consistent with all of our physical properties. We assume Nagata's[46] values for the enthalpy for the formation of a hydrogen bond.

To extend the analysis to alcohol mixtures, the cross-association parameters for dissimilar components are related through combining rules. Common combining rules that have been developed and tested[22,23,27,34,57-59] are variations of an arithmetic or geometric mean of the pure components' association energy and volume parameters. Kontogeorgis[38] outlines a number of these and provides a brief summary of work done in identifying the most accurate rules for various classes of mixtures. We find that Flory's polymerization theory and Wertheim's perturbation theory yield identical results for alcohols described with a 2B model, regardless of the combining rules used if Eq. (22) is maintained for the cross coefficients. Therefore, further 
exploration of the effect of the combining rules lies beyond the scope and intentions of this work and for alcohol mixtures, we use Suresh and Elliott's[22] combining rule given by:

$$
\Delta^{A_{i} B_{j}}=\Delta^{A_{j} B_{i}}=\sqrt{\Delta^{A_{i} B_{i}} \Delta^{A_{j} B_{j}}}
$$

\subsection{Numerical Equivalence of Chemical and Wertheim's Theory}

We implement the abovementioned approaches to provide numerical evidence for the equivalence of chemical theory and Wertheim's theory. Within the constraints discussed, we find that the two approaches produce exactly the same results for systems in which the associating components can be described by the $2 \mathrm{~B}$ association scheme. This is illustrated in Table 3 for two alcohol-inert systems at 30 and $80 \mathrm{~mol} \%$ of alcohol and one alcohol-alcohol system at $30 \mathrm{~mol} \%$ of methanol. Both theories yield the same monomer fractions and activity coefficients.

Table 3: Calculated parameters and intermediate values for Flory and Wertheim's theories for three systems. The reference density of methanol used for Campbell's theory calculations is $0.02440 \mathrm{~mol} / \mathrm{cm}^{3}$. For methanol + ethanol, the cross coefficient is $\Delta^{A_{1} D_{2}}=\Delta^{A_{2} D_{1}}=2559 \mathrm{~cm}^{3} / \mathrm{mol}$ or $\mathrm{K}_{\mathrm{C} 4, \mathrm{j}}=62.44$

\begin{tabular}{|c|c|c|c|c|c|c|c|}
\hline \multicolumn{2}{|c|}{ System } & \multicolumn{2}{|c|}{$\begin{array}{l}\text { Ethanol (1) + } \\
n \text {-heptane (2) }\end{array}$} & \multicolumn{2}{|c|}{$\begin{array}{c}\text { 1-Pentanol (1) + } \\
\text { n-heptane (2) }\end{array}$} & \multicolumn{2}{|c|}{$\begin{array}{c}\text { Methanol (1) + Ethanol } \\
\text { (2) }\end{array}$} \\
\hline \multicolumn{2}{|c|}{$x_{1}$} & 0.3 & 0.8 & 0.3 & 0.8 & \multicolumn{2}{|c|}{0.3} \\
\hline \multicolumn{2}{|c|}{$T(K)$} & 344.9 & 344.7 & 368.4 & 383.1 & \multicolumn{2}{|c|}{347.0} \\
\hline \multicolumn{2}{|c|}{ Component $i$} & 1 & 1 & 1 & 1 & 1 & 2 \\
\hline \multirow{7}{*}{$\begin{array}{c}\text { Campbell's } \\
\text { Chemical } \\
\text { Theory }\end{array}$} & $K_{C 4}$ & 67.37 & 67.65 & 53.60 & 40.30 & 60.76 & 64.16 \\
\hline & $\Psi$ & 0.2680 & 0.1790 & 0.2427 & 0.1419 & \multicolumn{2}{|c|}{0.1025} \\
\hline & $\Psi_{\mathrm{i}}^{0}$ & 0.0944 & 0.0942 & 0.0749 & 0.0849 & 0.1203 & 0.0966 \\
\hline & $C_{i, M}^{\prime}$ & 0.0101 & 0.0123 & 0.0119 & 0.0184 & 0.0043 & 0.0095 \\
\hline & $C_{i, M}^{\prime 0}$ & 0.0128 & 0.0128 & 0.0149 & 0.0192 & 0.0145 & 0.0134 \\
\hline & $x_{M} \frac{n_{0}}{n_{T}}$ & 0.0301 & 0.0230 & 0.0396 & 0.0527 & 0.0056 & 0.0124 \\
\hline & $\ln \gamma_{i}^{\text {chem }}$ & 0.7195 & 0.06100 & 0.5280 & 0.03109 & -0.002853 & -0.0004456 \\
\hline Wertheim's & $\begin{array}{c}\Delta^{A_{i} D_{i}} \\
\left(\mathrm{~cm}^{3} / \mathrm{mol}\right)\end{array}$ & 2761 & 2773 & 2197 & 1652 & 2490 & 2629 \\
\hline & $X^{A_{i}}=X^{D_{i}}$ & 0.3167 & 0.1696 & 0.3633 & 0.2566 & 0.1361 & 0.1330 \\
\hline
\end{tabular}




\begin{tabular}{|c|c|c|c|c|c|c|c|}
\hline & $x_{M} \frac{n_{0}}{n_{T}}$ & 0.0301 & 0.0230 & 0.0396 & 0.0527 & 0.0056 & 0.0124 \\
\cline { 2 - 7 } & $\ln \gamma_{i}^{\text {assoc }}$ & 0.9281 & 0.09320 & 0.5513 & 0.03344 & 0.02999 & 0.004305 \\
\cline { 2 - 8 } & $\ln \gamma_{i}^{\text {Flory }}$ & -0.2086 & -0.03220 & -0.02337 & -0.002351 & -0.03285 & -0.004751 \\
\cline { 2 - 7 } & $\ln \gamma_{i}^{\text {chem }}$ & 0.7195 & 0.06100 & 0.5280 & 0.03109 & -0.0028533 & -0.0004455 \\
\hline
\end{tabular}

These results prove that Campbell's observation of the similarities between his association model and Wertheim's perturbation theory are justified. Indeed, when combinatorial effects are added to the perturbation theory and the correct relationship between the association parameters of the two models is implemented, their mathematical forms become identical. Chapman's recognition that Wertheim's model gives the same distribution as Flory's model is also consistent with this work. Future work in this area will look at extending the analysis to components with multiple acceptors and donors.

\section{Conclusions}

In this work, we outline derivations for Wertheim's perturbation theory's activity coefficient model and chemical theory and compare the two models. The focus is for the $2 \mathrm{~B}$ scheme where each associating component has at one acceptor and one donor site per molecule such as alcoholinert and alcohol-alcohol mixtures. In these cases, the association strength $\Delta^{A_{i} B_{j}}$ in Wertheim's theory is found to be equal to the concentration based equilibrium constant $K_{C 1}$ used in Flory's polymerization theory. This elucidates the relationship between the two association models. Furthermore, we consider Campbell's implementation of chemical theory and show that the activity coefficient model is the sum of Flory's combinatorial term, calculated on a superficial basis, and the association contribution calculated with Wertheim's perturbation theory. The similarities drawn by Campbell between his theory and Wertheim's theory are therefore consistent with this work. The correlation between the association models also supports Chapman's understanding that the species distribution predicted by Flory's and Wertheim's models are identical. Finally, we provide in Eq. (7) an activity coefficient equation based on Wertheim's theory useful for common situations when the packing factor is universal and excess volume is zero. In Eqs. (A10) and (A14) we provide a new generalized expression for activity coefficients. 


\section{Acknowledgements}

This material is based upon work supported by the National Science Foundation under Grant No. 1603705. 


\section{List of Symbols}

\begin{tabular}{|c|c|}
\hline$a$ & interaction parameter of mixture \\
\hline$a_{i}$ & interaction parameter of species $i$ \\
\hline$a_{i j}$ & interaction parameter between species $i$ and $j$ \\
\hline A & Helmholtz energy \\
\hline$b$ & van der Waal size parameter of mixture \\
\hline$b_{i}$ & van der Waal size parameter of species $i$ \\
\hline$B^{\text {free }}$ & free contribution to the second virial coefficient \\
\hline$C_{i}$ & concentration of component $i$ (apparent unless accompanied by subscript T) \\
\hline$f$ & constant in Eq. (14) \\
\hline$g$ & function defined by Eq. (18) \\
\hline G & Gibbs energy \\
\hline$h$ & function defined by Eq.(A1) \\
\hline K & equilibrium constant \\
\hline$n$ & number of moles \\
\hline$n^{B_{i}, \text { free }}$ & number of nonbonded sites of type $B_{i}$ \\
\hline$n^{B_{i}, \text { total }}$ & total number of sites of type $B_{i}$ in solution \\
\hline$n^{*}$ & effective number of carbon atoms in an ether homomorph molecule \\
\hline$N_{B, i}$ & number of sites identical to site $i$ of type $B$ \\
\hline$P$ & pressure \\
\hline$P_{i}^{v a p}$ & vapor pressure of component $i$ \\
\hline$r_{i}$ & ratio of $\rho$ of methanol at $303.15 \mathrm{~K}$ and $\rho_{i}$ \\
\hline$R$ & universal gas constant \\
\hline$T$ & temperature \\
\hline$V_{i}$ & molar volume of pure component $i$ \\
\hline $\bar{V}$ & volume \\
\hline$x_{i}$ & liquid mole fraction of component $i$ (apparent unless accompanied by subscript $\mathrm{T}$ ) \\
\hline$X^{B_{i}}$ & fraction of sites identical to site $i$ of type $B$ that remain nonbonded at equilibrium \\
\hline $\bar{Z}$ & compressibility factor \\
\hline
\end{tabular}




\section{Greek Letters}

\begin{tabular}{|l|l|}
\hline$\alpha_{j, i}$ & function defined by Eq. (C2) \\
\hline$\gamma$ & activity coefficient \\
\hline$\Delta$ & Wertheim's association strength \\
\hline$\eta$ & packing fraction \\
\hline$\rho$ & molar density (apparent unless accompanied by subscript T) \\
\hline$\sigma$ & contact distance \\
\hline$\varphi_{i}$ & fugacity coefficient of pure component $i$ \\
\hline$\Phi_{i}$ & volume fraction of component or species $i$ (apparent unless accompanied by subscript T) \\
\hline$\Psi$ & total true dimensionless concentration of complexes (Campbell's work) \\
\hline$\Psi_{i}$ & true dimensionless concentration of all chains that end in $i$ (Campbell's work) \\
\hline
\end{tabular}

\section{Subscripts}

\begin{tabular}{|l|l|}
\hline 0 & pertaining to apparent species \\
\hline$a$ & activity based \\
\hline$A$ & pertaining to the alcohol \\
\hline$C$ & concentration based \\
\hline$D$ & dimer \\
\hline$h$ & hypothetical fluid in Brandani's work \\
\hline host & pertaining to component hosting the site \\
\hline$M$ & monomer \\
\hline$T$ & pertaining to true species \\
\hline$x$ & mole fraction based \\
\hline
\end{tabular}

\section{Superscripts}

\begin{tabular}{|l|l|}
\hline$\prime$ & Dimensionless (used for Campbell's concentration) \\
\hline$\circ$ & standard state property \\
\hline 0 & at purity \\
\hline assoc & association (Wertheim's) contribution \\
\hline attr & attractive contribution \\
\hline$B_{i} E_{j}$ & interaction between site $i$ of type $B$ and site $j$ of type $E$ \\
\hline
\end{tabular}




\begin{tabular}{|l|l|}
\hline chem & chemical theory \\
\hline comb & combinatorial contribution \\
\hline E & excess property \\
\hline Flory & Flory's theory \\
\hline ig & ideal gas \\
\hline L & pertaining to a liquid \\
\hline ref & pertaining to a reference state \\
\hline rep & repulsive contribution \\
\hline res & residual contribution \\
\hline
\end{tabular}

Special notation

\begin{tabular}{|c|c|}
\hline - as in $\underline{A}$ & extensive property \\
\hline as in $\bar{V}$ & partial molar property \\
\hline as in $\hat{\varphi}$ & property of component in a mixture \\
\hline
\end{tabular}




\section{Appendix A}

Following Michelsen and Hendricks[39] and to abbreviate notation, we define, at equilibrium,

$$
h=\sum_{\substack{\text { all site } \\ \text { types } B_{i}}} x_{B_{i} \text { host }} N_{B_{i}}\left(1-X^{B_{i}}\right)
$$

Elliott and Lira[40] show that:

$$
\begin{gathered}
Z^{\text {assoc }}=-0.5\left(1+\left(\frac{\partial \ln g}{\partial \ln \eta_{P}}\right)_{T, n}\right) h \\
\ln \hat{\varphi}_{k}^{\text {assoc }}=\sum_{\begin{array}{c}
\text { sites } B_{i} \\
\text { only on } k
\end{array}} N_{B_{i}} \ln X^{B_{i}}-0.5 n_{0}\left(\frac{\partial \ln g}{\partial n_{k}}\right)_{T, \underline{V}, n_{j \neq k}} h
\end{gathered}
$$

We will use these equations to calculate and simplify the equation for $\gamma^{\text {assoc }}$. By definition, Helmholtz energy is a natural function of temperature and volume:

$$
\underline{A}(\underline{V},\{n\}, T)
$$

For an activity coefficient, we need a derivative of Helmholtz energy at constant $T$ and $P$ [42]. At constant $T$,

$$
d \underline{A}=\left(\frac{\partial \underline{A}}{\partial \underline{V}}\right)_{T, n} d \underline{V}+\sum_{k}\left(\frac{\partial \underline{A}}{\partial n_{k}}\right)_{T, \underline{V}, n_{j \neq k}} d n_{k}
$$

The expansion rule gives

$$
\left(\frac{\partial \underline{A}}{\partial n_{k}}\right)_{T, P, n_{j \neq k}}=\left(\frac{\partial \underline{A}}{\partial \underline{V}}\right)_{T, n}\left(\frac{\partial \underline{V}}{\partial n_{k}}\right)_{T, P, n_{j \neq k}}+\left(\frac{\partial \underline{A}}{\partial n_{k}}\right)_{T, \underline{V}, n_{j \neq k}}
$$

Recalling that

$$
\frac{1}{R T}\left(\frac{\partial \underline{A}^{a s s o c}}{\partial n_{k}}\right)_{T, \underline{V}, n_{j \neq k}}=\ln \hat{\varphi}_{k}^{a s s o c}
$$

and

$$
\left(\frac{\partial \underline{A}^{a s s o c}}{\partial \underline{V}}\right)_{T, n}=-P^{a s s o c}
$$

And making the appropriate substitutions into Eq. (A6): 


$$
\frac{1}{R T}\left(\frac{\partial \underline{A}^{a s s o c}}{\partial n_{k}}\right)_{T, P, n_{j \neq k}}=-\frac{Z^{a s s o c}}{V} \bar{V}_{k}+\ln \hat{\varphi}_{k}^{a s s o c}
$$

Substituting Eqs. (A2 and A3) yields the universal equation:

$$
\begin{aligned}
\frac{1}{R T}\left(\frac{\partial \underline{A}^{a s s o c}}{\partial n_{k}}\right)_{T, P, n_{j \neq k}} & \\
= & \sum_{\begin{array}{c}
\text { sites } B_{i} \\
\text { only on } k
\end{array}} N_{B_{i}} \ln X^{B_{i}} \\
& +\frac{1}{2} h\left[\frac{\bar{V}_{k}}{V}\left(1+\left(\frac{\partial \ln g}{\partial \ln \eta_{P}}\right)_{T, n}\right)-n_{0}\left(\frac{\partial \ln g}{\partial n_{k}}\right)_{T, \underline{V}, n_{j \neq k}}\right]
\end{aligned}
$$

To simplify this further, we assume that $Z^{r e p}$ is given by the van der Waals equation. Applying Eq. (18) results in $g=1 /(1-b \rho)=\left(1 /\left(1-\eta_{P}\right)\right.$.

Eq. (A10) becomes:

$$
\begin{gathered}
\left(\frac{\partial \ln g}{\partial \ln \eta_{P}}\right)_{T, n}=\eta_{P} /\left(1-\eta_{P}\right) \\
n_{0}\left(\frac{\partial \ln g}{\partial n_{k}}\right)_{T, \underline{V}, n_{j \neq k}}=\frac{b_{k} \rho_{k}}{1-\eta_{P}}\left(\frac{V_{k}}{V}\right)
\end{gathered}
$$

$$
\frac{1}{R T}\left(\frac{\partial \underline{A}^{a s s o c}}{\partial n_{k}}\right)_{T, P, n_{j \neq k}}=\sum_{\begin{array}{c}
\text { sites } B_{i} \\
\text { only on } k
\end{array}} N_{B_{i}} \ln X^{B_{i}}+\frac{1}{2} h\left[\frac{\bar{V}_{k}}{V}\left(\frac{1}{1-\eta_{P}}\right)-\frac{V_{k}}{V}\left(\frac{b_{k} \rho_{k}}{1-\eta_{P}}\right)\right]
$$

If excess volume is zero and the packing factor is universal,

$$
\frac{1}{R T}\left(\frac{\partial \underline{A}^{a s s o c}}{\partial n_{k}}\right)_{T, P, n_{j \neq k}}=\sum_{\begin{array}{c}
\text { sites } B_{i} \\
\text { only on } k
\end{array}} N_{B_{i}} \ln X^{B_{i}}+\frac{1}{2}\left(\frac{\rho}{\rho_{k}}\right) \sum_{\begin{array}{c}
\text { all site } \\
\text { types } B_{i}
\end{array}} x_{B_{i} \text { host }} N_{B_{i}}\left(1-X^{B_{i}}\right)
$$

Using this equation, $\gamma^{a s s o c}$ can be calculated as:

$$
\gamma_{k}^{a s s o c}=\left.\frac{1}{R T}\left(\frac{\partial \underline{A}^{a s s o c}}{\partial n_{k}}\right)_{T, P, n_{j \neq k}}\right|_{\text {mix }}-\left.\frac{1}{R T}\left(\frac{\partial \underline{A}^{a s s o c}}{\partial n_{k}}\right)_{T, P, n_{j \neq k}}\right|_{\text {pure } k}
$$

This results in Eq. (7) in the text. Note that $x_{i} \rho / \rho_{k}=\Phi_{i}$ when excess volume is zero. 


\section{Appendix B}

Beginning with the fugacity coefficient relation, we substitute van der Waals equation of state:

$$
Z_{T}=\frac{P \underline{V}}{n_{T} R T}=1+\frac{b \rho_{T}}{1-b \rho_{T}}-\frac{a \rho_{T}}{R T}
$$

and its implementation into the Helmholtz departure equation:

$$
\frac{\left(\underline{A}-\underline{A}^{i g}\right)_{T, \underline{V}}}{n_{T} R T}=-\ln \left(1-b \rho_{T}\right)-\frac{a \rho_{T}}{R T}
$$

For a true associated species in a pure fluid:

$$
\ln \hat{\varphi}_{i}=-\ln \left(1-b \rho_{T}\right)+\frac{b_{i} \rho_{T}}{1-b \rho_{T}}-\frac{2 \rho_{T} \Sigma_{j} x_{T, j} a_{i j}}{R T}-\ln Z_{T}
$$

Applying Eq. (16) and (17), calculating Eq. (B3) for a monomer and a dimer gives:

$$
\ln \frac{\hat{\varphi}_{M}^{2}}{\hat{\varphi}_{D}}=-\ln \left(1-b \rho_{T}\right)-\ln Z_{T}
$$

or

$$
\frac{\hat{\varphi}_{D}}{\hat{\varphi}_{M}{ }^{2}}=Z_{T}\left(1-b \rho_{T}\right)=\frac{P \underline{V}\left(1-b \rho_{T}\right)}{n_{T} R T}=\frac{\hat{\varphi}_{i+1}}{\hat{\varphi}_{i} \hat{\varphi}_{M}}
$$




\section{Appendix C}

Here, we provide proof that $\Psi_{i}$ is the true concentration of all chains ending with $i$. Beginning with the definition of $\Psi_{i}$ in Campbell's[35] paper:

$$
\Psi_{i}=C_{i, 1}^{\prime}+\sum_{j} \alpha_{j, i}\left(C_{j, 1}^{\prime}+\sum_{k} \alpha_{k, j}\left(C_{k, 1}^{\prime}+\sum_{l} \alpha_{l, k}\left(C_{l, 1}^{\prime}+\cdots\right.\right.\right.
$$

where

$$
\alpha_{j, i}=K_{C 4, j i} C_{i, 1}^{\prime}
$$

Multiplying through Eq. (C1),

$$
\Psi_{i}=C_{i, 1}^{\prime}+\sum_{j} \alpha_{j, i} C_{j, 1}^{\prime}+\sum_{j} \alpha_{j, i} \sum_{k} \alpha_{k, j} C_{k, 1}^{\prime}+\sum_{j} \alpha_{j, i} \sum_{j} \alpha_{k, j} \sum_{l} \alpha_{l, k} C_{l, 1}^{\prime}+\cdots
$$

In Campbell's appendix C, the concentration of all chains of length $n$ ending in $i$ is given by:

$$
C_{i, n}=\sum_{j} \alpha_{j, i} C_{j, n-1}
$$

This equation provides a recursion for simplification. Recognizing equation C4 in the second, third, and fourth term on the right hand side of Eq. (C3) can be expressed as:

Then

$$
C_{i, 2}^{\prime}=\sum_{j} \alpha_{j, i} C_{j, 1}^{\prime} \text { and } C_{j, 2}^{\prime}=\sum_{k} \alpha_{k, j} C_{k, 1}^{\prime} \text { and } C_{k, 2}^{\prime}=\sum_{l} \alpha_{l, k} C_{l, 1}^{\prime}
$$

$$
\Psi_{i}=C_{i, 1}^{\prime}+C_{i, 2}^{\prime}+\sum_{j} \alpha_{j, i} C_{j, 2}^{\prime}+\sum_{j} \alpha_{j, i} \sum_{j} \alpha_{k, j} C_{k, 2}^{\prime}+\cdots
$$

Simplifying the third and fourth term on the right with Eq. (C4) again,

$$
\Psi_{i}=C_{i, 1}^{\prime}+C_{i, 2}^{\prime}+C_{i, 3}^{\prime}+\sum_{j} \alpha_{j, i} C_{j, 3}{ }^{\prime}+\cdots
$$

This procedure applied continuously to all sums in Eq. (C3) yields the result that $\Psi_{i}$ is also the true concentration of all chains that end in $i$.

$$
\Psi_{i}=C_{i, 1}^{\prime}+C_{i, 2}^{\prime}+C_{i, 3}^{\prime}+C_{i, 4}^{\prime}+\cdots=\sum_{j} C_{i, j}^{\prime}
$$




\section{References}

[1] G.K. Folas, G.M. Kontogeorgis, M.L. Michelsen, E.H. Stenby, Application of the cubicplus-association equation of state to mixtures with polar chemicals and high pressures, Ind. Eng. Chem. Res. 45 (2006) 1516-1526.

[2] A. Apelblat, The concept of associated solutions in historical development. Part 1 . The 1884-1984 period, J. Mol. Liq. 128 (2006) 1-31. doi:10.1016/j.molliq.2006.02.005.

[3] A. Apelblat, Erratum to "The concept of associated solutions in historical development. Part 1. The 1884-1984 period.,” J. Mol. Liq. 130 (2007) 133-162.

[4] F. Dolezalek, Zur Theorie der Binären Gemische und Konzentrierten Lösungen, Z Phys Chem. 64 (1908) 727-747.

[5] I. Nagata, K. Tamura, S. Tokuriki, Excess enthalpies and complex formation of acetonitrile with acetone, chloroform and benzene, Thermochim. Acta. 47 (1981) 315-331.

[6] I. Nagata, Y. Kawamura, Thermodynamics of Alcohol-Unassociated Active Component Liquid Mixtures, Chem. Eng. Sci. 34 (1979) 601-611.

[7] N.D. Coggeshall, E.L. Saier, Infrared Absorption Study of Hydrogen Bonding Equilibria, J. Am. Chem. Soc. 73 (1951) 5414-5418. doi:10.1021/ja01155a118.

[8] E.A. Guggenheim, Statistical thermodynamics of mixtures with zero energies of mixing, Proc. R. Soc. Lond. Ser. Math. Phys. Sci. 183 (1944) 203-212.

[9] P. Muthukumaran, R.L. Brinkley, R.B. Gupta, Lattice-fluid equation of state with hydrogen-bond cooperativity, Aiche J. 48 (2002) 386-392. doi:10.1002/aic.690480221.

[10] R.B. Gupta, R.L. Brinkley, Hydrogen-bond cooperativity in 1-alkanol plus n-alkane binary mixtures, Aiche J. 44 (1998) 207-213. doi:10.1002/aic.690440122.

[11] C. Panayiotou, M. Pantoula, E. Stefanis, I. Tsivintzelis, I.G. Economou, Nonrandom Hydrogen-Bonding Model of Fluids and Their Mixtures. 1. Pure Fluids, Ind. Eng. Chem. Res. 43 (2004) 6592-6606. doi:10.1021/ie040114+.

[12] C. Panayiotou, I. Tsivintzelis, I.G. Economou, Nonrandom Hydrogen-Bonding Model of Fluids and Their Mixtures. 2. Multicomponent Mixtures, Ind. Eng. Chem. Res. 46 (2007) 2628-2636. doi:10.1021/ie0612919.

[13] K.E. Gubbins, Perturbation Theories of the Thermodynamics of Polar and Associating Liquids: A Historical Perspective, Fluid Phase Equilibria. (2015) 1-15. doi:10.1016/j.fluid.2015.12.043.

[14] P.T. Cummings, G. Stell, Statistical mechanical models of chemical reactions, Mol. Phys. 51 (1984) 253-287. doi:10.1080/00268978400100191.

[15] G. Stell, Y. Zhou, Chemical association in simple models of molecular and ionic fluids, J. Chem. Phys. 91 (1989) 3618-3623. doi:10.1063/1.456894.

[16] Y. Zhou, G. Stell, Chemical association in simple models of molecular and ionic fluids. II. Thermodynamic properties, J. Chem. Phys. 96 (1992) 1504-1506. doi:10.1063/1.462872.

[17] M.S. Wertheim, Fluids with highly directional attractive forces.1. Statistical thermodynamics, J. Stat. Phys. 35 (1984) 19-34. doi:10.1007/bf01017362.

[18] M.S. Wertheim, Fluids with highly directional attractive forces. 2. Thermodynamic perturbation-theory and integral-equations, J. Stat. Phys. 35 (1984) 35-47. doi:10.1007/bf01017363.

[19] M.S. Wertheim, Fluids with highly directional attractive forces. 3. Multiple attraction sites, J. Stat. Phys. 42 (1986) 459-476. doi:10.1007/bf01127721. 
[20] M.S. Wertheim, Fluids with highly directional attractive forces. 4. Equilibrium polymerization, J. Stat. Phys. 42 (1986) 477-492. doi:10.1007/bf01127722.

[21] J.R. Elliott, S.J. Suresh, M.D. Donohue, A simple equation of state for nonspherical and associating molecules, Ind. Eng. Chem. Res. 29 (1990) 1476-1485. doi:10.1021/ie00103a057.

[22] S.J. Suresh, J.R. Elliott, Multiphase Equilibrium Analysis via a Generalized Equation of State for Associating Mixtures, Ind. Eng. Chem. Res. 31 (1992) 2783-2794.

[23] G.M. Kontogeorgis, E.C. Voutsas, I. V. Yakoumis, D.P. Tassios, An Equation of State for Associating Fluids, Ind. Eng. Chem. Res. 35 (1996) 4310-4318. doi:10.1021/ie9600203.

[24] I. V Yakoumis, G.M. Kontogeorgis, E.C. Voutsas, E.M. Hendriks, D.P. Tassios, Prediction of Phase Equilibria in Binary Aqueous Systems Containing Alkanes, Cycloalkanes, and Alkenes with the Cubic-plus-Association Equation of State, Ind. Eng. Chem. Res. 37 (1998) 4175-4182.

[25] W.G. Chapman, K.E. Gubbins, G. Jackson, M. Radosz, New reference equation of state for associating liquids, Ind. Eng. Chem. Res. 29 (1990) 1709-1721. doi:10.1021/ie00104a021.

[26] J. Gross, G. Sadowski, Perturbed-Chain SAFT: An Equation of State Based on a Perturbation Theory for Chain Molecules, Ind. Eng. Chem. Res. 40 (2001) 1244-1260. doi:10.1021/ie0003887.

[27] Y.-H. Fu, S.I. Sandler, A Simplified SAFT Equation of State for Associating Compounds and Mixtures, Ind. Eng. Chem. Res. 34 (1995) 1897-1909. doi:10.1021/ie00044a042.

[28] S.H. Huang, M. Radosz, Equation of state for small, large, polydisperse, and associating molecules, Ind. Eng. Chem. Res. 29 (1990) 2284-2294. doi:10.1021/ie00107a014.

[29] A. Gil-Villegas, A. Galindo, P.J. Whitehead, S.J. Mills, G. Jackson, A.N. Burgess, Statistical associating fluid theory for chain molecules with attractive potentials of variable range, J. Chem. Phys. 106 (1997) 4168-4186. doi:10.1063/1.473101.

[30] T. Kraska, K.E. Gubbins, Phase Equilibria Calculations with a Modified SAFT Equation of State. 1. Pure Alkanes, Alkanols, and Water, Ind. Eng. Chem. Res. 35 (1996) 4727-4737. doi:10.1021/ie9602320.

[31] I.G. Economou, M.D. Donohue, Chemical, quasi-chemical and perturbation theories for associating fluids, AIChE J. 37 (1991) 1875-1894. doi:10.1002/aic.690371212.

[32] J.P. Wolbach, S.I. Sandler, Using molecular orbital calculations to describe the phase behavior of hydrogen-bonding fluids, Ind. Eng. Chem. Res. 36 (1997) 4041-4051. doi:10.1021/ie9607255.

[33] E.M. Hendriks, J. Walsh, A.R.D. van Bergen, A general approach to association using cluster partition functions, J. Stat. Phys. 87 (1997) 1287-1306. doi:10.1007/BF02181285.

[34] J.P. Wolbach, S.I. Sandler, Using molecular orbital calculations to describe the phase behavior of cross-associating mixtures, Ind. Eng. Chem. Res. 37 (1998) 2917-2928. doi:10.1021/ie970781l.

[35] S.W. Campbell, Chemical theory for mixtures containing any number of alcohols, Fluid Phase Equilibria. 102 (1994) 61-84.

[36] J.M. Prausnitz, F.W. Tavares, Thermodynamics of Fluid-Phase Equilibria for Standard Chemical Engineering Operations, AIChE J. 50 (2004) 739-761. doi:10.1002/aic.10069.

[37] M.L. Michelsen, Robust and efficient solution procedures for association models, Ind. Eng. Chem. Res. 45 (2006) 8449-8453. doi:10.1021/ie060029x.

[38] G.M. Kontogeorgis, G.K. Folas, Thermodynamic Models for Industrial Applications, John Wiley \& Sons, 2010. 
[39] M.L. Michelsen, E.M. Hendriks, Physical properties from association models, Fluid Phase Equilibria. 180 (2001) 165-174. doi:10.1016/S0378-3812(01)00344-2.

[40] J.R. Elliott, C.T. Lira, Introductory Chemical Engineering Thermodynamics, International Edition, 2nd ed., Pearson Education, Upper Saddle River, NJ, 2012.

[41] J.R. Elliott, C.T. Lira, Introductory Chemical Engineering Thermodynamics, 2nd ed., Errata, (n.d.). http://chethermo.net/errata (accessed July 13, 2016).

[42] Y. Fu, S.I. Sandler, H. Orbey, A Modified UNIQUAC Model That Includes Hydrogen Bonding, Ind. Eng. Chem. Res. 34 (1995) 4351-4363.

[43] P.J. Flory, Thermodynamics of Heterogeneous Polymers and Their Solutions, J. Chem. Phys. 12 (1944) 425. doi:10.1063/1.1723887.

[44] H. Renon, J.M. Prausnitz, On the thermodynamics of alcohol-hydrocarbon solutions, Chem. Eng. Sci. 22 (1967) 299-307. doi:10.1016/0009-2509(67)80116-7.

[45] H. Renon, J.M. Prausnitz, On the thermodynamics of alcohol-hydrocarbon solutions. Errata., Chem. Eng. Sci. 22 (1967) 1891. doi:10.1016/0009-2509(67)80223-9.

[46] I. Nagata, Thermodynamics of alcohol solutions. Phase equilibria and excess molar enthalpies of mixtures containing two alcohols, Thermochim. Acta. 107 (1986) 199-217.

[47] A. Nath, E. Bender, On the thermodynamics of associated solutions. I. An analytical method for determining the enthalpy and entropy of association and equilibrium constant for pure liquid substances, Fluid Phase Equilibria. 7 (1981) 275-287. doi:10.1016/03783812(81)80012-X.

[48] I. Nagata, K. Gotoh, Thermodynamics of alcohol solutions, Thermochim. Acta. 258 (1995) 77-107.

[49] V. Brandani, A continuous linear association model for determining the enthalpy of hydrogen-bond formation and the equilibrium association constant for pure hydrogenbonded liquids, Fluid Phase Equilibria. 12 (1983) 87-104.

[50] V. Brandani, F. Evangelista, The UNIQUAC associated-solution theory: vapor-liquid equilibria of binary systems containing one associating and one inert or active component, Fluid Phase Equilibria. 17 (1984) 281-302.

[52] R.A. Heidemann, J.M. Prausnitz, A van der Waals-type equation of state for fluids with associating molecules., Proc. Natl. Acad. Sci. U. S. A. 73 (1976) 1773-6.

[52] A.G. Pradhan, V.R. Bhethanabotla, S.W. Campbell, Vapor-liquid equilibrium data for ethanol-n-heptane-1-propanol and ethanol-n-heptane-2-propanol and their interpretation by a simple association model, Fluid Phase Equilibria. 84 (1993) 183-206. doi:10.1016/03783812(93)85123-4.

[53] G.M. Lobien, J.M. Prausnitz, Correlation for the ratio of limiting activity coefficients for binary liquid mixtures, Fluid Phase Equilibria. 8 (1982) 149-160. doi:10.1016/03783812(82)80032-0.

[54] W.A. Fouad, L. Wang, A. Haghmoradi, S.K. Gupta, W.G. Chapman, Understanding the Thermodynamics of Hydrogen Bonding in Alcohol-Containing Mixtures: Self Association, J. Phys. Chem. B. 119 (2015) 14086-14101. doi:10.1021/acs.jpcb.5b08285.

[55] J.G. Hayden, J.P. O’Connell, A generalized method for predicting second virial coefficients, Ind. Eng. Chem. Process Des. Dev. 14 (1975) 209-216. doi:10.1021/i260055a003.

[56] D. Ambrose, J.H. Ellender, C.H.S. Sprake, R. Townsend, Thermodynamic properties of organic oxygen compounds: Vapour pressures of some ethers, J. Chem. Thermodyn. 8 (1976) 165-178. 
[57] A. Grenner, G.M. Kontogeorgis, N. von Solms, M.L. Michelsen, Application of PC-SAFT to glycol containing systems - PC-SAFT towards a predictive approach, Fluid Phase Equilibria. 261 (2007) 248-257. doi:10.1016/j.fluid.2007.04.025.

[58] J. Suresh, E.J. Beckman, Prediction of liquid-liquid equilibria in ternary mixtures from binary data, Fluid Phase Equilibria. 99 (1994) 219-240. doi:10.1016/0378-3812(94)800332.

[59] E.C. Voutsas, I.V. Yakoumis, D.P. Tassios, Prediction of phase equilibria in water/alcohol/alkane systems, Fluid Phase Equilibria. 158-160 (1999) 151-163. 